OPEN ACCESS

Edited by:

Vijai Kumar Gupta,

National University of Ireland, Galway,

Ireland

Reviewed by:

Marcos Sergio Toledo,

Universidade Federal de São Paulo,

Brazi

Praveen Rao Juvvadi,

Duke University Medical Center, USA

*Correspondence:

Yueming Jiang

ymjiang@scbg.ac.cn

tThese authors contributed equally to this work.

Specialty section: This article was submitted to Fungi and Their Interactions,

a section of the journal

Frontiers in Microbiology

Received: 22 February 2016

Accepted: 20 June 2016

Published: 29 June 2016

Citation:

$L i T$, Jian $Q$, Chen F, Wang $Y$,

Gong L, Duan X, Yang $B$ and

Jiang $Y$ (2016) Influence of Butylated

Hydroxyanisole on the Growth,

Hyphal Morphology,

and the Biosynthesis of Fumonisins

in Fusarium proliferatum.

Front. Microbiol. 7:1038.

doi: 10.3389/fmicb.2016.01038

\section{Influence of Butylated Hydroxyanisole on the Growth, Hyphal Morphology, and the Biosynthesis of Fumonisins in Fusarium proliferatum}

\author{
Taotao Li',2†, Qijie Jian',2†, Feng Chen ${ }^{3}$, Yong Wang ${ }^{4}$, Liang Gong' ${ }^{1}$, Xuewu Duan', \\ Bao Yang ${ }^{1}$ and Yueming Jiang ${ }^{1 *}$ \\ 1 Key Laboratory of Plant Resource Conservation and Sustainable Utilization, Guangdong Provincial Key Laboratory of \\ Applied Botany, South China Botanical Garden, Chinese Academy of Sciences, Guangzhou, China, ${ }^{2}$ University of Chinese \\ Academy of Sciences, Beijing, China, ${ }^{3}$ Department of Food, Nutrition and Packaging Sciences, Clemson University, \\ Clemson, SC, USA, ${ }^{4}$ Zhong Shan Entry-Exit Inspection and Quarantine Bureau, Zhong Shan, China
}

Fusarium proliferatum as a common fungus pathogen in foods can produce toxic fumonisins, which can cause animal diseases and increase risks of human cancers. On contrary, butylated hydroxyanisole (BHA) as a synthetic antioxidant offers a clue for preventing growth of fungal species and inhibiting production of mycotoxins. Unfortunately, information of the inhibitory mechanism of $\mathrm{BHA}$ on Fusarium species is still limited. In this study, influence of BHA treatment on growth and inhibition of fumonisin production in relation to the expression of the fumonisin biosynthesis-related genes of the $F$. proliferatum ZYF was investigated, which revealed that $\mathrm{BHA}$ had a negative influence on growth and fumonisin production of $F$. proliferatum. To further elucidate the mechanism of BHA on the growth of $F$. proliferatum, scanning electron microscopy (SEM) and transmission electron microscopy (TEM) were used to examine the F. proliferatum hyphae. The BHA treatment induced the loss of cytoplasm and cellular constituents, as well as distortion of mycelia, but it did not directly degrade the fumonisin. Furthermore, the BHA treatment markedly inhibited the expressions of FUM1 (a polyketide synthase encoding gene) and FUM8 (an aminotransferase encoding gene) genes, which resulted in the depression of metabolic pathway of $F$. proliferatum. The transcriptional analyses of the FUM1 and FUM8 genes confirmed a correlation between the fumonisin production and its gene expression. This study provided some insights into mechanisms of production of fumonisin and feasible prevention to reduce fumonisin contamination in favor of human and animal health.

Keywords: fumonisin, Fusarium proliferatum, butylated hydroxyanisole, gene expression, mycelia

\section{INTRODUCTION}

Fusarium proliferatum that is commonly found in fruits such as banana (Anthony et al., 2004) and mango (Zhan et al., 2010) is a fungal pathogen, of which infection could induce a rapid deterioration of fruits ( $\mathrm{Li}$ et al., 2012). In addition, many mycotoxins, such as fumonisins, moniliformin, beauvericin, fusaproliferin, fusaric acid, and bikaverin, can be produced by 
F. proliferatum. Li et al. (2012) reported that the F. proliferatum isolated from banana peel produced a large amount of fusaric acid (5-butylpicolinic acid). Besides, F. proliferatum isolated from banana fruit could yield a large amount of fumonisin B1 (FB1), rather than other mycotoxins present in corn and rice cultures (Jimenez et al., 1997). Due to the genetic variation, different F. proliferatum variants can produce different mycotoxins in food and fruits. Regardless of the significant progress on development of rapid, sensitive and robust assays to detect the presence of mycotoxins, there lacks enough efforts to reduce or even eliminate the presence of the mycotoxins in foods and feeds (Yang et al., 2014). Thus, it is worthy to investigate effective and practical means to inhibit the growth of F. proliferatum so as to reduce relevant mycotoxins in food.

Fumonisins can be produced by many Fusarium species including $F$. proliferatum, and act as one of the most harmful fungal toxins, which can cause animal diseases and human esophageal cancer (Marasas, 2001). Among these fumonisins, B-type fumonisins are the most toxic. FB1 accounts for almost 70\%, while FB2 and FB3 make up about $10-20 \%$ of the total fumonisin content (Nelson et al., 1993). In recent years, some studies have reported the presence of fumonisin B1 or B2 in some fruits (Karbancioglu-Güler and Heperkan, 2008; Senyuva and Gilbert, 2008). In addition, it was found that FUM1 (a polyketide synthase gene) encoding the $\beta$-ketoacyl synthase domain catalyzed the synthesis of the linear polyketide of fumonisins (Fanelli et al., 2012), while FUM8 (an aminotransferase encoding gene) involved in the last step of fumonisin biosynthesis (Seo et al., 2001). Moreover, substantial information has been accumulated in regards of the key gene expression of the fumonisin biosynthesis by the Fusarium species (Lopez-Errasquin et al., 2007). On the other hand, although some factors to affect the fumonisin production and FUM gene expression of $F$. proliferatum have been reported (Samapundo et al., 2005; Kohut et al., 2009; Marín et al., 2010), little information is available on their relationship. Therefore, it is necessary for further investigation to understand and elucidate the mechanisms in controlling fumonisin biosynthesis.

Some compounds could inhibit the growth of F. proliferatum, but they might not be efficient in reducing production of toxins (Torres et al., 2003). For instance, a recent study reported that cinnamon oil could be a promising candidate in controlling FB1 production in corn products (Xing et al., 2014a). In addition, a synthetic antioxidant butylated hydroxyanisole (BHA) is commonly used in industrial processing for protection of cereals, grain products, cooking oils, canned goods, snacks, and many other foods (Fung et al., 1977). BHA was found to have inhibitory effects on growth, sporulation, pigmentation, and toxigenesis of Aspergilius flavus (Fung et al., 1977). Other researches indicated that BHA could inhibit Fusarium verticillioides and $F$. proliferatum populations and then reduce fumonisin production on maize grain (Torres et al., 2003; Farnochi et al., 2005). Recently, the role of BHA in controlling Fusarium growth and fumonisin production was well reviewed by Alberts et al. (2016). Our preliminary study also indicated that BHA could significantly inhibit the growth of F. proliferatum and its fusaric acid production in the culture medium. However, different species or strains could have different responses to environment factors (Jimenez et al., 2003). Nevertheless, these studies have not established a link between the fumonisin production and the expression level of fumonisin biosynthetic genes. To the best of our knowledge, the effects of BHA on the expression of the fumonisin biosynthesis-related genes and anti-fungal mechanism of $F$. proliferatum have not been investigated. Therefore, the major objective of this study was to investigate the influence of BHA on the growth, morphology and fumonisin (FB1 and FB2) production, as well as FUM gene expression by F. proliferatum. Besides, this study is expected to take early preventive actions to reduce the fumonisin contamination in an effort to protect human and animal health. On the other hand, better understanding of the inhibitive mechanism of BHA against pathogens might provide new clue for finding new targeted action of novel fungicides.

\section{MATERIALS AND METHODS}

\section{Fungal Strain and Growth Condition}

Fusarium proliferatum ZYF was isolated from carambola and then stored in $50 \%$ glycerol at $-80^{\circ} \mathrm{C}$. The strain was grown for 7 days at $28^{\circ} \mathrm{C}$ on potato dextrose agar (PDA) (Oxoid, Basingstoke, Hampshire, England) in 90-mm Petri dishes. Six small agar disks $(5 \mathrm{~mm})$ with mycelia were taken by punching bear from colony edge and then transferred to the Czapek's broth $(\mathrm{CB})$ medium $\left(3.0 \mathrm{~g} / \mathrm{L} \mathrm{NaNO}_{3}, 1.0 \mathrm{~g} / \mathrm{L} \mathrm{K}_{2} \mathrm{HPO}_{4}, 0.5 \mathrm{~g} / \mathrm{L}\right.$ $\mathrm{MgSO}_{4} \cdot 7 \mathrm{H}_{2} \mathrm{O}, 0.5 \mathrm{~g} / \mathrm{L} \mathrm{KCl}, 0.01 \mathrm{~g} / \mathrm{L} \mathrm{FeSO}_{4}$, and $30 \mathrm{~g} / \mathrm{L}$ sucrose, sterilized previously at $121^{\circ} \mathrm{C}$ for $20 \mathrm{~min}$ ) in the presence and absence of $0.5 \mathrm{mM}$ BHA (Aladdin, Shanghai, China). In this study, a concentration of $0.5 \mathrm{mM}$ of BHA was chosen based on our previous experiments since this concentration could significantly inhibit the growth of $F$. proliferatum. The conical flask containing $200 \mathrm{ml}$ of the above-mentioned cultures was incubated at $28^{\circ} \mathrm{C}$ for 10 days in an orbital shaker at $200 \mathrm{rpm}$. Growth curves were expressed in an arbitrary unit by the method of Kohut et al. (2009). Experiments were repeated at least three times.

\section{Scanning Electron Microscopy and Transmission Electron Microscopy}

For the scanning electron microscopy (SEM) and transmission electron microscopy (TEM) experiments, the ZYF strains were placed into the potato dextrose agar in $90-\mathrm{mm}$ Petri dishes in the presence and absence of $0.5 \mathrm{mM} \mathrm{BHA}$, and then maintained for 10 days at $28^{\circ} \mathrm{C}$. The diameters of the colonies were measured in two directions at a right angle to analyze the growth and morphology of ZYF according to the method of Fanelli et al. (2012). The samples for the SEM and TEM experiments were conducted after 10 days of fungal growth.

For the SEM examination, several segments (about 5$10 \mathrm{~mm}$ ) of the cultures grown on the PDA plates were cut and immobilized in vials containing $2.5 \%$ glutaraldehyde and $2 \%$ paraformaldehyde for at least $48 \mathrm{~h}$ at $4^{\circ} \mathrm{C}$. These samples were then washed twice with $0.1 \mathrm{M}$ phosphate buffer saline, fixed with 
osmic acid for $2.5 \mathrm{~h}$, washed again with the $0.1 \mathrm{M}$ phosphate buffer saline and then dried sequentially for $10 \mathrm{~min}$ in a series of ethanol $(30,50,70,80,90$, and $100 \%$, v/v). Following the dehydration, the samples were washed with threitol for three times (10 min each) and then frozen in a lyophilizer. Finally, these samples were coated for $180 \mathrm{~s}$ with $20 \mathrm{~mA}$ gold-palladium electroplating in a sputter coater (JFC-1600, JEOL, Japan). All samples were examined by a JSM-6360 LV SEM (JEOL, Japan) at $15 \mathrm{kV}$.

The same 10-day-old fungal cultures on the PDA plates were used for the TEM observations. The dehydration step was adopted as same as that for the SEM, then the samples were successively treated with epoxypropane, epoxypropane:resin (EPON 812, SPI, USA) (3:1), epoxypropane:resin (1:1), and epoxypropane:resin (1:3) for $1 \mathrm{~h}$. Finally, these samples were soaked in $100 \%$ resin overnight and then embedded into the resin for $36 \mathrm{~h}$ at $60^{\circ} \mathrm{C}$. Ultrathin samples were made by ultramicrotome (Leica UC6, Germany) and mounted on copper grids. After being stained with $2 \%$ uranyl acetate and lead citrate for $30 \mathrm{~min}$, respectively, these samples were examined carefully using a transmission electron microscope (JEM-1010) (JEOL, Japan) at an accelerating voltage of $100 \mathrm{kV}$.

\section{Fumonisin Extraction and LC-MS/MS Analysis}

Fumonisin was extracted by the method of Seefelde and Humpf (2002). The culture filtrates $(50 \mathrm{ml})$ were collected at the 7 - and 10-day and then extracted in a $100 \mathrm{ml}$ solution of methanol/water (3:1, v/v) (HPLC-grade, ANPEL, Shanghai, China) using a double-layer filter paper. After modifying the $\mathrm{pH}$ to 5.8-6.5, the filtrate was purified with a SAX (strong anion exchange) column (500 mg, $6 \mathrm{ml}$, ANPEL, Shanghai). The SAX column was activated previously with $8 \mathrm{ml}$ methanol and methanol/water $(3: 1, \mathrm{v} / \mathrm{v})$ successively. The filtrate $(8 \mathrm{ml})$ through the column was purified with $8 \mathrm{ml}$ methanol/water $(3: 1, \mathrm{v} / \mathrm{v})$ and methanol successively. Finally, the column was eluted with $10 \mathrm{ml}$ methanol containing $1 \%$ acetic acid. The obtained eluant was concentrated to an appropriate volume by $\mathrm{N}_{2}$ purge, and then methanol/water $(60: 40, \mathrm{v} / \mathrm{v})$ was added to a final volume of $1 \mathrm{ml}$. The solution was filtered through a $0.22-\mu \mathrm{m}$ Millipore membrane filter prior to the ultra performance liquid chromatography (UPLC)-MS/MS analysis.

The analyses of FB1 and FB2 were conducted by an AB-SCIEX TRIPLE QUAD ${ }^{\mathrm{TM}} 5500$ UPLC-MS/MS system (AB SCIEX, USA). The obtained sample $(10 \mu \mathrm{L})$ was injected onto an Ekspert 100 UPLC column $(\mathrm{C} 18$ column, $100 \times 2.1 \mathrm{~mm}, 3 \mu \mathrm{m}$ particle size, Thermo, USA). An optimized gradient of mobile phase (A: acetonitrile and B: $5 \mathrm{mM}$ ammonium acetate) was used (Table 1). $\mathrm{N}_{2}$ was used as a nebulizing air at nebulizer temperature of $450^{\circ} \mathrm{C}$. Positive ionization was selected for mass spectrometric (MS) detection. A multiple reaction monitoring (MRM) function was employed for quantification, with the fragment ions at $\mathrm{m} / z$ 722.5 for FB1 and 706.4 for FB2, respectively. The MS conditions were optimized for both FB1 and FB2, including declustering potential $(60 \mathrm{~V})$, entrance potential $(10 \mathrm{~V})$, collision exit potential $(14 \mathrm{~V})$, collision energy $(50 \mathrm{~V})$, and ion spray voltage $(5500 \mathrm{~V})$.
TABLE 1 | Ultra performance liquid chromatography (UPLC) gradient program and pump time.

\begin{tabular}{lccc}
\hline Time (min) & Flow rate (ml/min) & Fraction A (\%) & Fraction B (\%) \\
\hline $0-0.5$ & 0.4 & 10 & 90 \\
$0.5-8$ & 0.4 & $10-50$ & $90-50$ \\
$8-8.5$ & 0.4 & $50-10$ & $50-90$ \\
$8.5-9$ & 0.4 & 10 & 90 \\
\hline
\end{tabular}

The detection limit of FB1 and FB2 was $0.1 \mathrm{ng} \mathrm{ml}^{-1}$. The additional parameters of the analyses of FB1 and FB2 are given in Supplementary Table S1.

\section{RNA Isolation, Reverse Transcription and Real-Time q-PCR}

For RNA isolation, the mycelia of 10-day-old ZYF grown in $\mathrm{CB}$ were collected. The total RNA was extracted using the Hipure Fungal RNA Mini Kit (Magen, China), according to the manufacturer's instructions, and then stored at $-80^{\circ} \mathrm{C}$. The first strand cDNA was synthesized using the PrimeScript ${ }^{\mathrm{TM}} \mathrm{RT}$ Master Mix (TAKARA-RR036A, Dalian, China) subjected to RTPCR amplification. Real-time PCR assays were used to quantify the FUM1 and FUM8 expressions in F. proliferatum strain using the primer pairs described by Kohut et al. (2009), which is listed in Supplementary Table S2.

SYBR Premix Ex Taq ${ }^{\mathrm{TM}} \operatorname{mix}$ (TaKaRa, Dalian, China) was used as a reaction mixture by adding $10.0 \mu \mathrm{l}$ of SYBR Premix Ex Taq ${ }^{\mathrm{TM}}, 0.4 \mu \mathrm{l}$ of PCR forward primer $(10 \mu \mathrm{M}), 0.4 \mu \mathrm{l}$ of PCR reverse primer $(10 \mu \mathrm{M}), 0.4 \mu \mathrm{l}$ of ROX reference dyeII and $2 \mu \mathrm{l}(20 \mathrm{ng})$ of cDNA in a final volume of $20 \mu \mathrm{l}$. The real-time PCR was performed with a 7500 Fast Real-Time PCR System (Applied Biosystems, Foster City, CA, USA). The 7500 Fast System Software 2.0.1 was used to analyze data in fluorescent intensity. Amplification conditions were kept for $3 \mathrm{~min}$ at $95^{\circ} \mathrm{C}$, followed by 40 cycles at $95^{\circ} \mathrm{C}$ for $5 \mathrm{~s}, 60^{\circ} \mathrm{C}$ for $5 \mathrm{~s}$ and $72^{\circ} \mathrm{C}$ for $34 \mathrm{~s}$. The comparative $\mathrm{Ct}\left({ }^{\Delta}{ }^{\Delta} \mathrm{CT}\right)$ method was performed to determine the relative change in the FUM1 and FUM8 expressions.

\section{Release of Cellular Materials from F. proliferatum}

The method of Paul et al. (2011) was used to measure the release of cellular materials. After F. proliferatum ZYF was cultured for 10 days in the PDB and then the mycelia were collected by centrifugation at $4000 \times g$ for $20 \mathrm{~min}$, washed three times and resuspended in $50 \mathrm{ml}$ of $0.1 \mathrm{M}$ phosphate buffer saline ( $\mathrm{pH} 7.0$ ). The suspensions in the presence and absence of $0.5 \mathrm{mM}$ BHA were incubated with agitation in an environmental incubator shaker for $0,30,60$, and $120 \mathrm{~min}$ at $28^{\circ} \mathrm{C}$. Samples $(2 \mathrm{ml})$ was collected, then centrifuged at $12,000 \times g$ for $2 \mathrm{~min}$ and finally $1 \mathrm{ml}$ of the supernatant was used to determine the concentration of the released materials.

\section{Effect of BHA on FB1 Degradation}

In order to investigate whether BHA could degrade FB1 directly, FB1 was added to acetonitrile/water $(1: 1, \mathrm{v} / \mathrm{v})$ resulting in a 
A

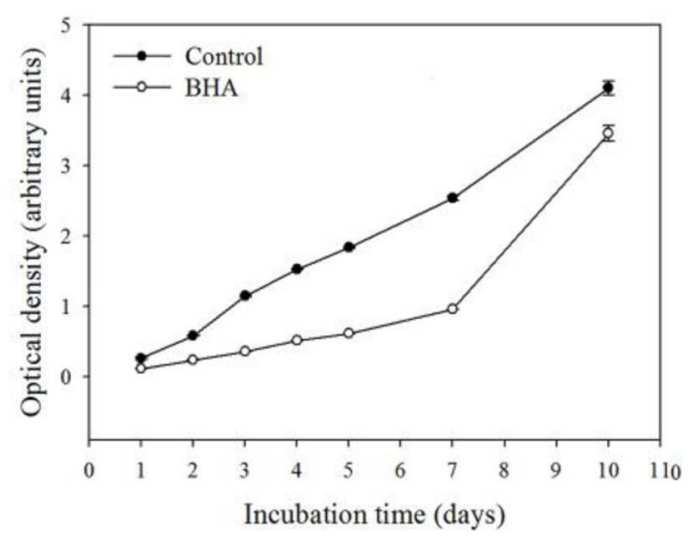

B

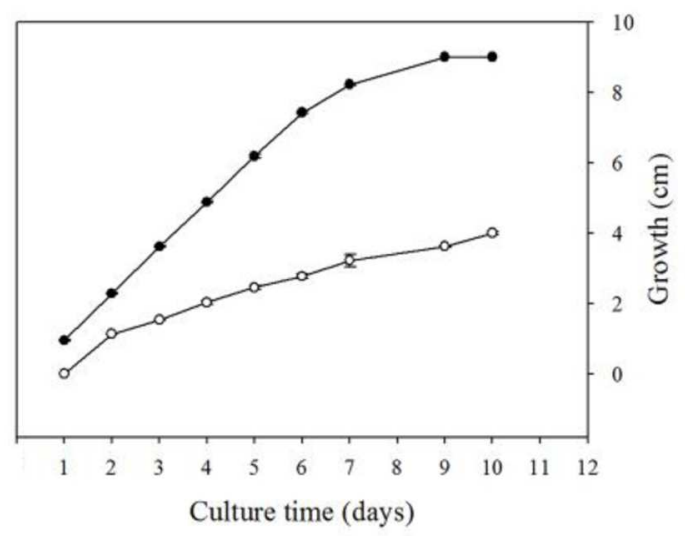

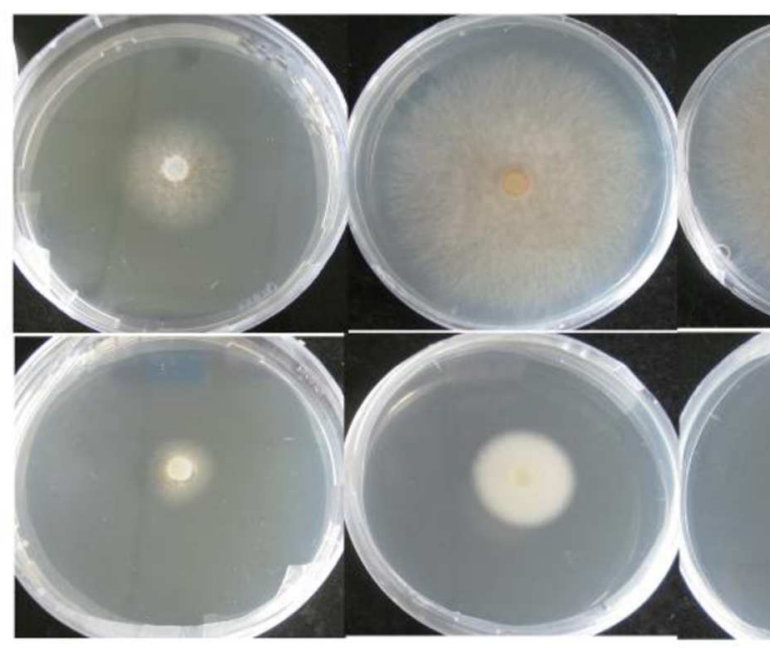

$3 d$

$5 d$

$7 \mathrm{~d}$

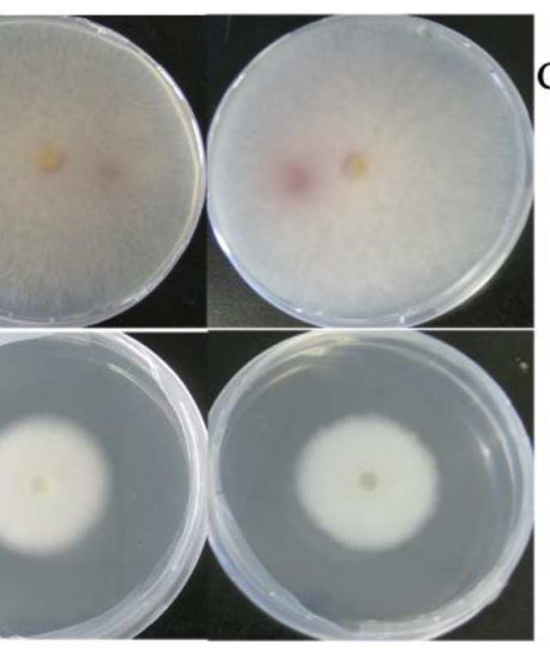

\section{BHA}

FIGURE 1 | Effect of BHA treatment on growth rates of Fusarium proliferatum ZYF incubation in Czapek's broth (CB) medium (A) and PDA plate (B,C). Values of optical densities were expressed in arbitrary units while diameters of the colonies were expressed as $\mathrm{cm}$. Vertical bars indicated standard errors $(n=3)$. Experiments were repeated three times with the same results.

concentration of $1 \mu \mathrm{g} / \mathrm{ml}$ in the presence and absence of $0.5 \mathrm{mM}$ BHA, according to the method of Xing et al. (2014a). The sample was shaken for $48 \mathrm{~h}$ at $200 \mathrm{rpm}$ and $25^{\circ} \mathrm{C}$, extracted and detected by UPLC-MS/MS described above. The degradation experiment was also preceded in the $\mathrm{CB}$ medium. FB1 was added into $100 \mathrm{ml}$ of $\mathrm{CB}$ in the presence and absence of $0.5 \mathrm{mM}$ BHA and then incubated at $28^{\circ} \mathrm{C}$ for $48 \mathrm{~h}$ with shaking at $200 \mathrm{rpm}$. FB1 was extracted and detected as the above-mentioned method.

\section{Statistical Analysis}

The SPSS 16.0 software (SPSS Inc., Chicago, IL, USA) was used for the statistical data analysis. The significant differences of growth rate and release of cellular materials from $F$. proliferatum among different culture time were analyzed by one-way analysis of variance (ANOVA). One-sample $t$-tests were used to compare the fumonisin production and FUM gene expression between the control and BHA-treated samples. The relationships between FUM gene expression and fumonisin production were evaluated using the Pearson correlation coefficients. The statistical significance was set at $p<0.05$.

\section{RESULTS}

\section{Growth Assessment}

It was observed that BHA significantly $(p<0.01)$ inhibited the growth of $F$. proliferatum ZYF both in the CB medium (Figure 1A) and PDA plate (Figure 1B). The inhibitory effect took place during the whole culturing period although a lag phase appeared at the late stage when $F$. proliferatum was grown on the PDA without $0.5 \mathrm{mM}$ BHA, due to the petri dishes restriction $(90 \mathrm{~mm}$ ) (Figures 1B,C). Interestingly, the growth rate of $F$. proliferatum in the CB medium increased at the late stage in the BHA-treated sample, but the growth of F. proliferatum was 

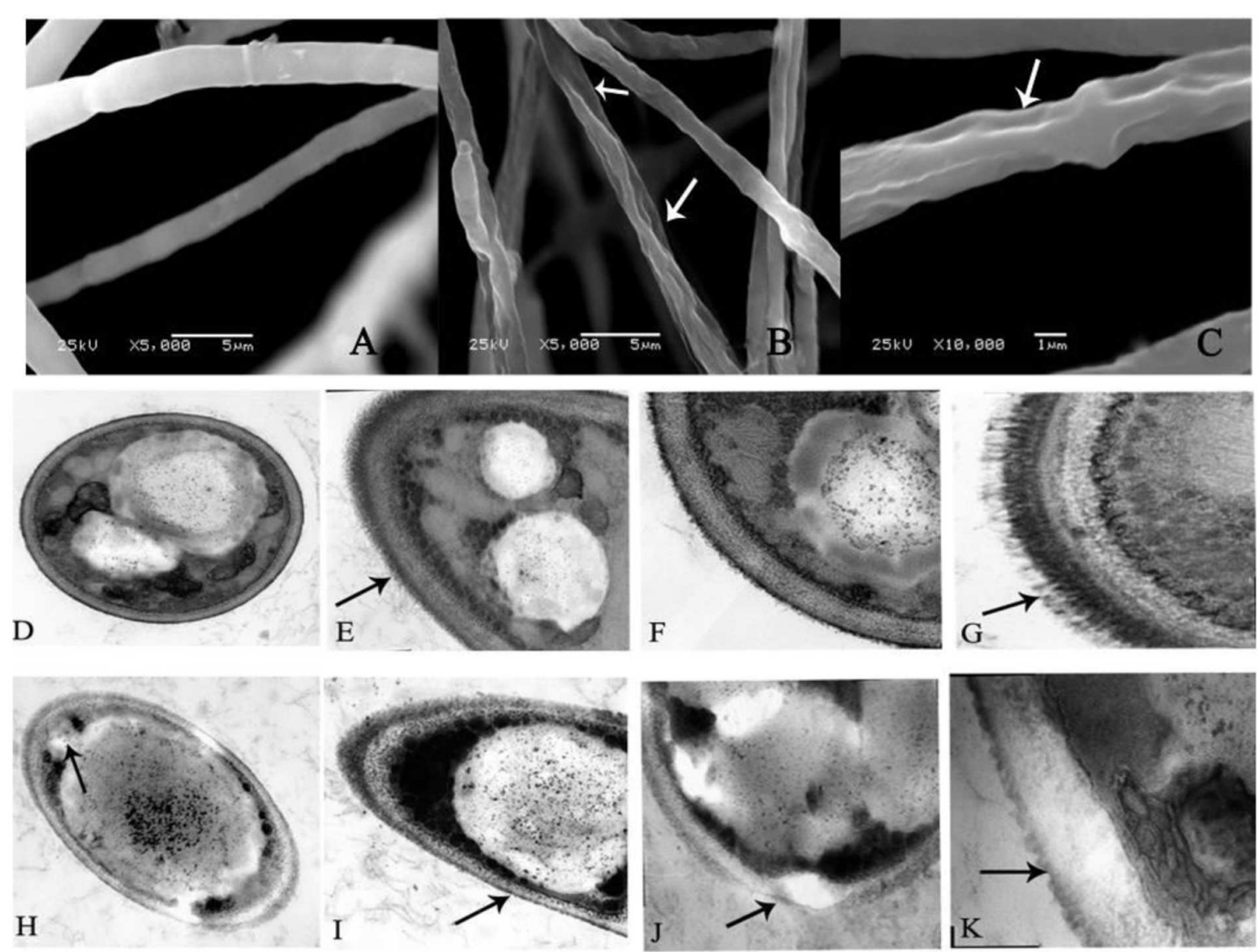

FIGURE 2 | Scanning electron microscopy (SEM) (A-C) and transmission electron microscopy (TEM) (D-K) images of F. proliferatum ZYF. (A) Mycelia of non-butylated hydroxyanisole (non-BHA)-treated (control) F. proliferatum ZYF after 10 days of culture; (B,C) The BHA-treated F. proliferatum ZYF after 10 days of culture. (D-G) Mycelia of non-BHA-treated (control) F. proliferatum ZYF after 10 days of culture (20, 30, 50, and 150×, respectively). (H-K) Mycelia of the BHA-treated $F$. proliferatum ZYF after 10 days of culture $(20,30,50$, and $150 \times$, respectively). Arrows referred to the morphologic changes in the hyphae.

still inhibited by the BHA treatment compared with that of the control. In comparison with the growth, slight changes in the morphology of the colonies were observed (Figure 1C).

\section{Scanning Electron Microscopy and Transmission Electron Microscopy}

As shown in Figure 2A, the fungus grown on the PDA plate in the absence of $0.5 \mathrm{mM}$ BHA showed normal, tubular, regular and homogrnous hyphae. On the contrary, the mycelia of $F$. proliferatum ZYF treated with $0.5 \mathrm{mM} \mathrm{BHA}$ showed significant changes in the hyphal morphology. The mycelium was shown in swelled and slender appearance while the swelled mycelium was malformed, indicating the collapsed cells were caused by lack of cytoplasm (Figure 2B), and had depressed hyphal surfaces (Figure 2C). Craters were also observed on the cell walls.

To further explain the inhibitory mechanism caused by the BHA treatment, the ultrastructure of the $F$. proliferatum ZYF was investigated by TEM. The cells of F. proliferatum ZYF in the absence of $0.5 \mathrm{mM}$ BHA showed intact cell structure (Figure 2D) and thick cell wall (Figures 2E,F), while the filiform was regular and thick (Figure 2G). On the contrary, the BHA treatment clearly induced turbulence of cellular structure and plenty of transparent inclusion with wide vacuoles in the cell center and large lipid globules beside the cellular walls (Figures $\mathbf{2 H}-\mathbf{K}$ ). Furthermore, the treatment remarkably resulted in disruption (Figure 2J) and alteration in cell walls (Figure 2I). Meanwhile, cell membrane damage of $F$. proliferatum ZYF was also observed (Figure $\mathbf{2 H}$ ), resulting in obvious loss of cytoplasm and release of cellular materials. Additionally, the filiform became irregular and thin (Figure 2K).

\section{Effect of BHA on Fumonisin Production and FB1 Degradation}

Both FB1 and FB2 were obtained from the $\mathrm{CB}$ culture in the presence and the absence of $0.5 \mathrm{mM} B H A$. The results of the UPLC-MS/MS quantification are presented in Figures 3A,B while the MS spectra for FB 1 and FB 2 are given in Supplementary Figure S1. After 10 days of culturing, the amount of FB1 was $44.53 \pm 0.29 \mathrm{ng} / \mathrm{ml}$ for the control and 
A

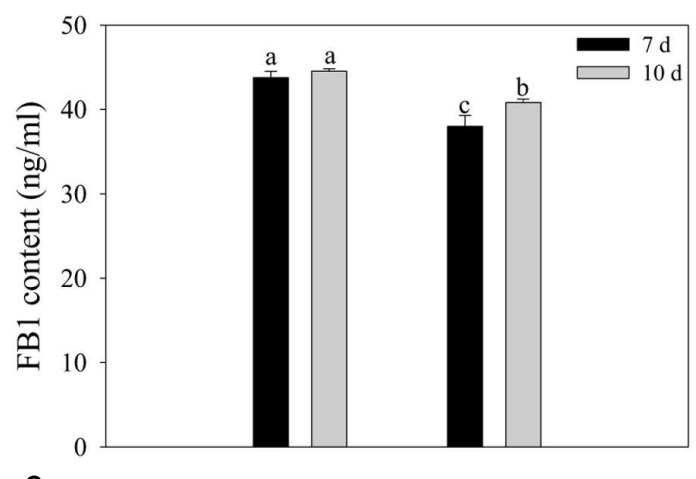

C

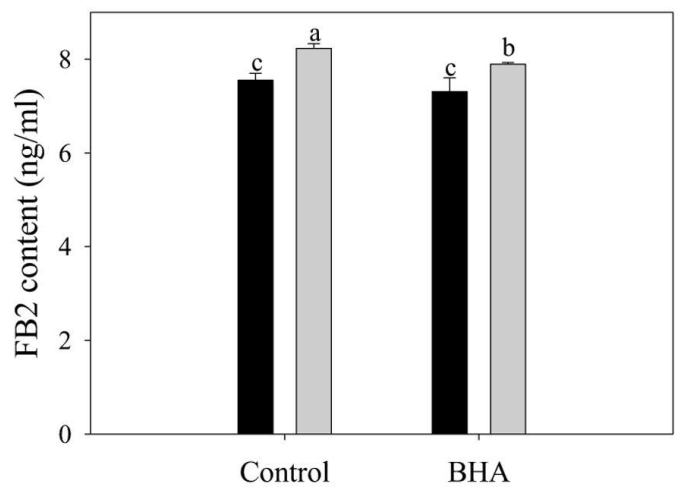

B

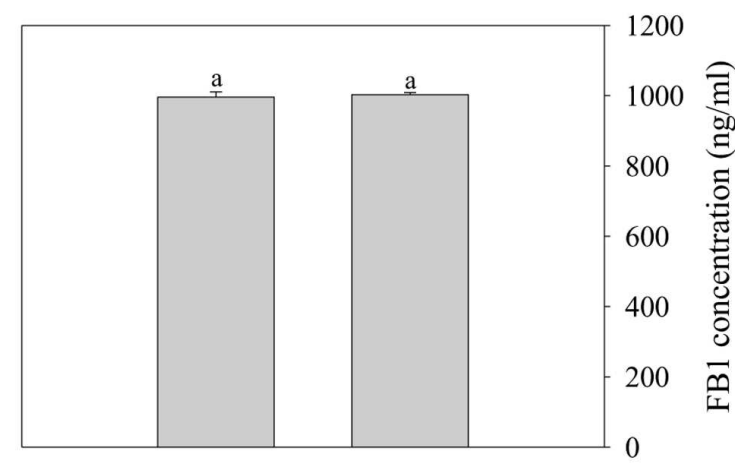

D

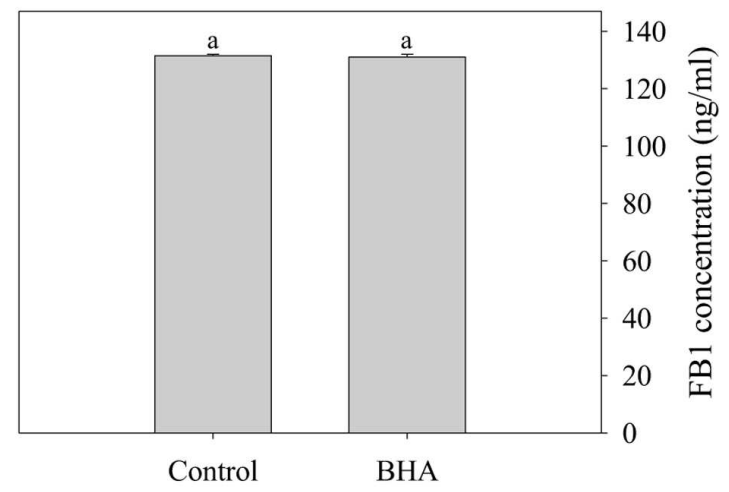

FIGURE 3 | Effects of BHA on extracellular production of FB1 (A) and FB2 (B) of $F$. proliferatum ZYF and the FB1 degradation when FB1 was incubated in acetonitrile/water (C) and CB media (D). Vertical bars indicated standard errors of three independent experiments.

$40.83 \pm 0.40 \mathrm{ng} / \mathrm{ml}$ for the BHA-treated sample. For FB2, the contents in the control and BHA-treated samples were $8.13 \pm 0.10$ and $7.89 \pm 0.04 \mathrm{ng} / \mathrm{ml}$, respectively. Our results indicated clearly that the contents of FB1 and FB2 of the BHA-treated sample of $F$. proliferatum ZYF were significantly $(p<0.05)$ lower than those of the control sample after 10 days of culture. In order to explain further the effect of BHA on the synthesis of fumonisin, the production levels of FB1 and FB2 after 7 days of culture in the $\mathrm{CB}$ medium were determined. As shown in Figures 3A,B, BHA treatment significantly $(p<0.05)$ reduced the content of $\mathrm{FB} 1$ while had no significant effect on FB2 content after 7 days of culture. Additionally, BHA treatment had no direct effect on the FB1 degradation in this study (Figures 3C,D). After $48 \mathrm{~h}$ of incubation in acetonitrile/water $(1: 1, v / v)$ solution, no significant difference was observed in regards of the FB1 concentration, with $996.00 \pm 14.69 \mathrm{ng} / \mathrm{ml}$ and $1003.00 \pm 5.72 \mathrm{ng} / \mathrm{ml}$ for the control and BHA-treated samples, respectively, while the content of FB1 was $131.50 \pm 0.50 \mathrm{ng} / \mathrm{ml}$ and $131.00 \pm 1.00 \mathrm{ng} / \mathrm{ml}$ for the control the BHA-treated samples in the CB medium, respectively.

\section{Expressions of FUM1 and FUM8}

The expression analyses of FUM1 and FUM8 are shown in Figures $4 \mathrm{~A}, \mathrm{~B}$. Compared with the genes in the control sample, the expressions of FUM1 and FUM8 genes were reduced significantly $(p<0.01)$ after the BHA treatment. The expression levels of FUM1 and FUM8 of the control sample were more than 4 and 2 folds higher than the BHA-treated sample, respectively. Furthermore, both FUM1 and FUM8 expressions exhibited significant $(p<0.01)$ correlations with the fumonisin contents in the control and BHA-treated samples (Figure 4C).

\section{Release of Cellular Contents}

Based on the absorbance value at $260 \mathrm{~nm}$, the release of cellular materials from $F$. proliferatum ZYF are presented in Figure 5. BHA treatment increased significantly $(p<0.01)$ the release of cellular materials during the whole culture period, which could account for the growth inhibition of $F$. proliferatum ZYF.

\section{DISCUSSION}

Fusarium proliferatum can cause the diseases of some economically important fruits after harvest. Moreover, fumonisin production by the $F$. proliferatum can have a serious effect on human and animal health. With an increasing concern on food safety, many researchers focus on understanding of the molecular mechanism involved in fumonisin production in order to develop efficient strategic solutions to reduce the fumonisin contamination in fruits. Velluti et al. (2003) reported that cinnamon oil had an inhibitory effect on the 
A

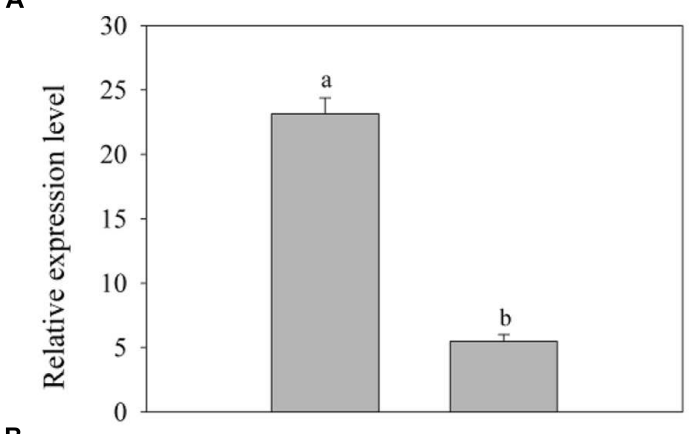

B
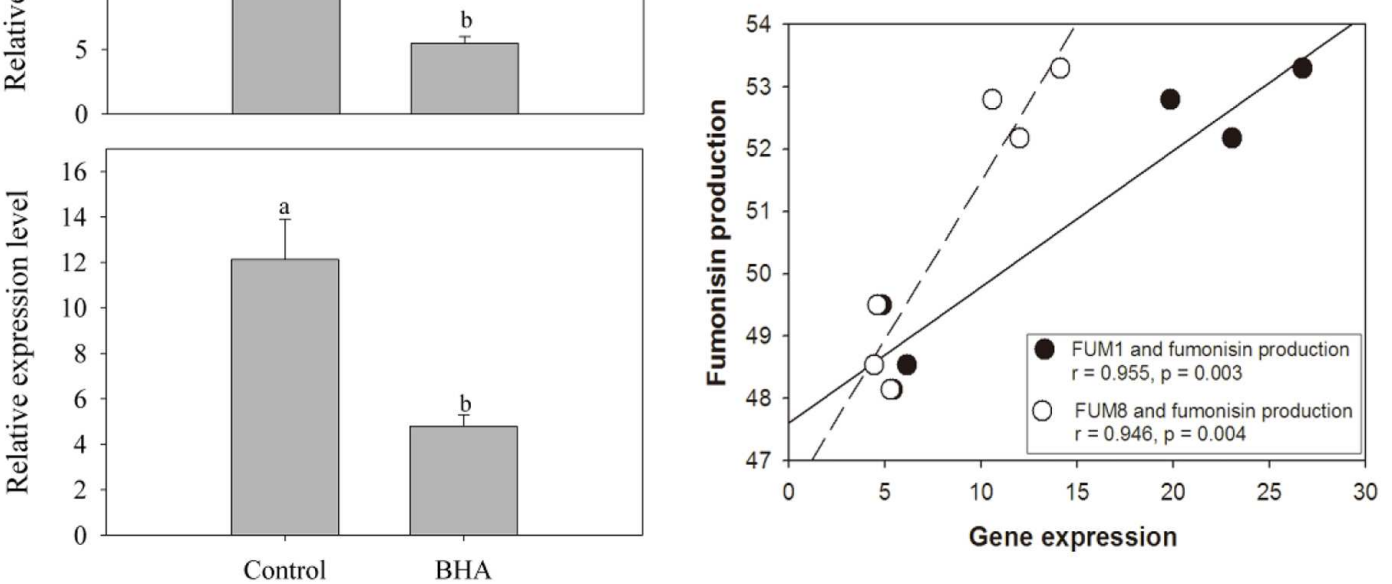

FIGURE 4 | The expression levels of FUM1 (A) and FUM8 (B) of F. proliferatum ZYF, and a correlation between fumonisin production and FUM gene expression (C). Induction of FUM genes was monitored by real-time PCR. Data calculated by using the ${ }^{\Delta \Delta} \mathrm{CT}$ method were expressed as relative units. The data were means of three independent repetitions. Vertical bars indicated standard errors.

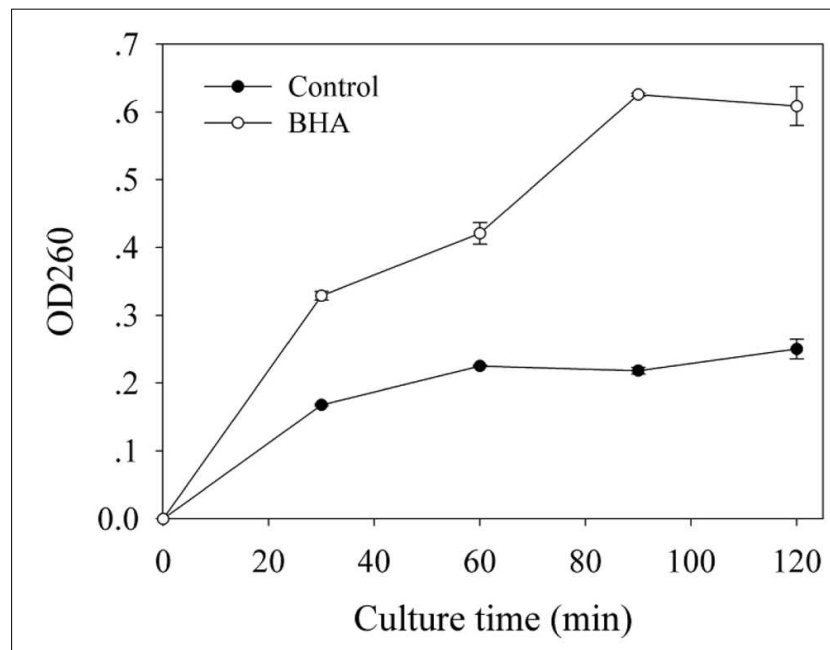

FIGURE 5 | Influence of BHA on release of cell materials. Data were presented as means \pm standard errors $(n=3)$.

F. proliferatum growth. On other hand, light, nutritional and environmental factors were reported to have different effects on the growth and fumonisin production of $F$. proliferatum (Kohut et al., 2009; Marín et al., 2010; Fanelli et al., 2012). The present study indicated that the BHA treatment could also inhibit the growth and reduced the fumonisin production and the gene expression of $F$. proliferatum ZYF, and the role of BHA treatment in inhibiting $\mathrm{FB1}$ production can be indirect.

Butylated hydroxyanisole was proven to be able to effectively inhibit the mycelium growth too (Torres et al., 2003; Farnochi et al., 2005). Fung et al. (1977) hypothesized that BHA as a phenolic compound might interact with cell membrane proteins to cause disruption of the membrane structure. Recently, Tao et al. (2014) indicated that the inhibition of the mycelium growth of Penicillium italicum after a citral treatment was due to the membrane damage. In the present study, BHA treatment can effectively inhibit the mycelium growth of $F$. proliferatum both in solid and liquid media (Figure 1). However, there was a lag phase when $F$. proliferatum was cultured on the PDA plate in the absence of $0.5 \mathrm{mM} \mathrm{BHA}$. In fact, after 7 days of culture, $F$. proliferatum in the absence of $\mathrm{BHA}$ grew to reach the edge of the Petri dishes (Figures 1B,C). To further monitor the growth of $F$. proliferatum on the PDA plate in the presence of BHA, the experiment was extended to 10 days. Thus, due to the restriction of the Petri dishes, slowed growth of $F$. proliferatum in the absence of BHA was observed while the BHA-treated F. proliferatum appeared to increase at the late culture stage (710 days). Similar phenomenon was also reported in Aspergillus spp. and other Fusarium species (Farnochi et al., 2005). Moreover, the growth of some fungi can be influenced by the interaction factors, such as antioxidant dose, water potential and temperature (Passone et al., 2012). Additionally, the growth of F. proliferatum could be influenced by different environmental factors, such as oxygen and nutrition. In the present study, it could be suggested 
that slow growth of $F$. proliferatum by the BHA treatment at the early stage might reduce the oxygen and nutrition consumption which might contribute to the cell growth of the BHA-treated $F$. proliferatum at the later stage. However, these suggestions need further confirmation in the future study.

The SEM observation further confirmed the significant change in morphological structure of $F$. proliferatum in the presence of BHA. The mycelia appeared swelled and collapsed (Figures 2B,C). This result was in agreement with the report that $F$. verticillioides exhibited the disruption of mycelia in the presence of essential oils (Xing et al., 2014b). Furthermore, $F$. proliferatum in the absence of BHA showed intact mycelia with normal structure, endoplasmic reticulum, and vacuoles within the cytoplasm, but the BHA-treated mycelia exhibited significant alterations in the hyphae including plasma membrane disruption (Figure 2H), thin cell walls (Figure 2I) or loss of integrity and rigidity of cell walls (Figure 2J). Irregular filiform of the BHAtreated $F$. proliferatum was also observed (Figure 2K). Previous study reported that the ultrastructure of fungi could be modified after the essential oil and citral treatments (Khan and Ahmad, 2011; Tao et al., 2014; Xing et al., 2014b), such as damages to cell walls, membranes and cytoplasmic contents. Additionally, loss of cellular materials confirmed further the membrane damage of F. proliferatum by the BHA treatment (Figure 5). Bajpai et al. (2013) reported that loss of cellular components could result from the irreversible damage of the cytoplasmic and plasma membranes.

FUM1 as the first gene identified in the fumonisin biosynthesis encodes a polyketide synthase which can catalyze the synthesis of the linear polyketide of fumonisins (Fanelli et al., 2012). FUM1 expression can be induced and repressed by $\mathrm{NaCl}$ at low and high concentrations (Jurado et al., 2008). For F. proliferatum, FUM1 transcription was induced under nonionic water stress at $15-35^{\circ} \mathrm{C}$ in $F$. proliferatum culture (Lazzaro et al., 2012a). FUM8 as an aminotransferase gene is transcriptionally regulated also (Seo et al., 2001). Although Susca et al. (2010) reported that the presence/absence of FUM8 was not correlated with FB2 production. However, FB1 production of $F$. proliferatum was associated with the expression level of FUM8 under the N-stress condition (Kohut et al., 2009). In this study, fumonisin concentrations measured during culture of $F$. proliferatum ZYF paralleled with the expression levels of FUM1 and FUM8 (Figures 3A,B, and 4A,B). Moreover, a relationship existed between expression levels for two genes and fumonisin concentrations. The BHA treatment reduced significantly $(p<0.01)$ the relative expressions of FUM1 and FUM8 (Figures $\mathbf{4 A , B}$ ). It is also worthy of mention that, although the growth of the BHA-treated $F$. proliferatum recovered between 7 and 10 days with a continuous increase of the fumonisin production, the content of fumonisin in the BHA-treated cells were still lower than that in control sample (Figures 3A,B). These results indicated the defect in the synthesis of fumonisins in the presence of BHA. To further clarify the influence of BHA on the FB1 biosynthesis, the FB1 degradation experiment was performed. It was found that the BHA treatment had no direct effect on the degradation of FB1 (Figures 3C,D), which suggested that the influence of BHA appeared to be only at the transcriptional period. The results were in agreement with the previous reports of Lazzaro et al. (2012b) and Lozano-Ojalvo et al. (2013), who reported that the biosynthesis of mycotoxin was regulated at a genetic level. Therefore, our results suggested that reduced expression of the FUM1 and FUM8 genes might be involved in the decrease in fumonisin content by BHA treatment. However, it is worthy to note that FUM1 and FUM8 were reduced by $70-80 \%$ while only $8 \%$ and $3 \%$ decrease in FB1 and FB2 contents was observed after the BHA treatment. Similarly, previous research also indicated that the inhibition rates of aflatoxins production and aflD gene expression were rather different in Aspergillus flavus RCP08108 (Passone et al., 2012). In this study, we cautiously postulated that inhibition of FUM1 and FUM8 expression might contribute partially to the decrease of FB1 and FB2 contents. Besides the possible functional importance of FUM1 and FUM8 genes in the inhibition of fumonisin production by BHA treatment, other genes might be also regulated by the treatment and involved in the fumonisin backbone modification and $\mathrm{ABC}$ transport during the biosynthesis of fumonisin (Proctor et al., 2003). In this context, it is necessary for further studies using RNASeq-based technique to find more genes which might be involved in the genetic regulations by the BHA treatment. For example, the analysis of the expression of FUM19, which encodes an $\mathrm{ABC}$ transporter and was previously correlated to fumonisin production, would enhance to verify the role of $\mathrm{BHA}$ in the biosynthesis and transport of fumonisins after the BHA treatment. Moreover, further work by applying bio-omics methods, such as proteomics and transcriptomics, might be able to explain more the mechanism of the inhibitory effect of BHA on the fumonisin production.

\section{AUTHOR CONTRIBUTIONS}

TL and YJ conceived and designed the study. TL, QJ, YW, LG, $\mathrm{XD}$, and $\mathrm{BY}$ performed the experiments and analyzed the data. TL, YJ, and FC drafted and revised the manuscript. All authors participated in the interpretation of data of the manuscript. All authors approved the submission and publication for all aspects of the work.

\section{ACKNOWLEDGMENTS}

This work was supported by the National Natural Science Foundation of China (Grant Nos. 31229004 and 31401593), Key Project Program of Chinese Academy of Sciences (No. KSZDEW-Z-021-3-3), and Science and Technology Planning Project of Guangdong Province (Nos. 2014A040401067).

\section{SUPPLEMENTARY MATERIAL}

The Supplementary Material for this article can be found online at: http://journal.frontiersin.org/article/10.3389/fmicb.2016. 01038 


\section{REFERENCES}

Alberts, J. F., Van Zyl, W. H., and Gelderblom, W. C. (2016). Biologically based methods for control of fumonisin-producing Fusarium species and reduction of the fumonisins. Front. Microbiol. 7:548. doi: 10.3389/fmicb.2016. 00548

Anthony, S. A. K., Dayananda, R., Wijeratnam, S. W., and Arambewela, L. (2004). Fungal pathogens associated with banana fruit in Sri Lanka, and their treatment with essential oils. Mycopathologia 157, 91-97. doi: 10.1023/B:MYCO.0000012226.95628.99

Bajpai, V. K., Sharma, A., and Baek, K. H. (2013). Antibacterial mode of action of Cudrania tricuspidata fruit essential oil, affecting membrane permeability and surface characteristics of food-borne pathogens. Food Control 32, 582-590. doi: 10.1016/j.foodcont.2013.01.032

Fanelli, F., Schmidt-Heydt, M., Haidukowski, M., Geisen, R., Logrieco, A., and Mulè, G. (2012). Influence of light on growth, fumonisin biosynthesis and FUM1 gene expression by Fusarium proliferatum. Int. J. Food Microbiol. 153, 148-153. doi: 10.1016/j.ijfoodmicro.2011.10.031

Farnochi, M. C., Torres, A. M., Magan, N., and Chulze, S. N. (2005). Effect of antioxidants and competing mycoflora on Fusarium verticillioides and F. proliferatum populations and fumonisin production on maize grain. J. Stored Prod. Res. 41, 211-219. doi: 10.1016/j.jspr.2004.03.004

Fung, D. Y. C., Taylor, S., and Kahan, J. (1977). Effects of butylated hydroxyanisole (BHA) and butylated hydroxytoluene (BHT) on growth and aflatoxin production of Aspergillus Flavus. J. Food Safety 1, 39-51. doi: 10.1111/j.17454565.1977.tb00258.x

Jimenez, M., Huerta, T., and Mateo, R. (1997). Mycotoxin production by Fusarium species isolated from bananas. Appl. Environ. Microbiol. 63, 364-369.

Jimenez, M., Mateo, J. J., Hinojo, M. J., and Mateo, R. (2003). Sugars and amino acids as factors affecting the synthesis of fumonisins in liquid cultures by isolates of the Gibberella fujikuroi complex. Int. J. Food Microbiol. 89, 185-193. doi: 10.1016/S0168-1605(03)00120-X

Jurado, M., Marín, P., Magan, N., and González-Jaén, M. T. (2008). Relationship between solute and matric potential stress, temperature, growth and Fum1 gene expression in two Fusarium verticillioides strains from Spain. Appl. Environ. Microbiol. 74, 2032-2036. doi: 10.1128/AEM. 02337-07

Karbancioglu-Güler, F., and Heperkan, D. (2008). Natural occurrence of ochratoxin a in dried figs. Anal. Chim. Acta 617, 32-36. doi: 10.1016/j.aca.2008.01.009

Khan, M. S., and Ahmad, I. (2011). In vitro antifungal, anti-elastase and antikeratinase activity of essential oils of Cinnamomum-, Syzygiumand Cymbopogon species against Aspergillus fumigates and Trichophyton rubrum. Phytomedicine 19, 48-55. doi: 10.1016/j.phymed.2011. 07.005

Kohut, G., Ádám, A. L., Fazekas, B., and Hornok, L. (2009). N-starvation stress induced FUM gene expression and fumonisin production is mediated via the HOG-type MAPK pathway in Fusarium proliferatum. Int. J. Food Microbiol. 130, 65-69. doi: 10.1016/j.ijfoodmicro.2009.01.002

Lazzaro, I., Busman, M., Battilani, P., and Butchko, R. A. E. (2012a). FUM and BIK gene expression contribute to describe fumonisin and bikaverin synthesis in Fusarium verticillioides. Int. J. Food Microbiol. 160, 94-98. doi: 10.1016/j.ijfoodmicro.2012.10.004

Lazzaro, I., Susca, A., Mule, G., Ritieni, A., Ferracane, R., Marocco, A., et al. (2012b). Effects of temperature and water activity on FUM2 and FUM21 gene expression and fumonisin B production in Fusarium verticillioides. Eur. J. Plant Pathol. 134, 685-695. doi: 10.1007/s10658-012$0045-y$

Li, J., Jiang, G., Yang, B., Dong, X., Feng, L., Lin, S., et al. (2012). A luminescent bacterium assay of fusaric acid produced by Fusarium proliferatum from banana. Anal. Bioanal. Chem. 402, 1347-1354. doi: 10.1007/s00216-011$5546-6$

Lopez-Errasquin, E., Vazquez, C., Jimenez, M., and Gonzalez-Jaen, M. T. (2007). Real-Time RT-PCR assay to quantify the expression of fuml and fum19 genes from the fumonisin-producing Fusarium verticillioides. J. Microbiol. Methods 68, 312-317. doi: 10.1016/j.mimet.2006. 09.007
Lozano-Ojalvo, D., Rodríguez, A., Bernáldez, V., Córdoba, J. J., and Rodríguez, M. (2013). Influence of temperature and substrate conditions on the omt-1 gene expression of Aspergillus parasiticus in relation to its aflatoxin production. Int. J. Food Microbiol. 166, 263-269. doi: 10.1016/j.ijfoodmicro.2013. 07.011

Marasas, W. F. O. (2001). Discovery and occurrence of the fumonisins: a historical perspective. Environ. Health Perspect. 109, 239-243. doi: 10.2307/34 35014

Marín, P., Magan, N., Vázquez, C., and González-Jaén, M. T. (2010). Differential effect of environmental conditions on the growth and regulation of the fumonisin biosynthetic gene FUM1 in the maize pathogens and fumonisin producers Fusarium verticillioides and Fusarium proliferatum. FEMS Microbiol. Ecol. 73, 303-311. doi: 10.1111/j.1574-6941.2010.00894.x

Nelson, P. E., Desjardins, A. E., and Plattner, R. D. (1993). Fumonisins, mycotoxins produced by Fusarium species: biology, chemistry, and significance. Annu. Rev. Phytopathol. 31, 233-252. doi: 10.1146/annurev.py.31.090193. 001313

Passone, M. A., Cristina Rosso, L., and Etcheverry, M. (2012). Influence of sub-lethal antioxidant doses, water potential and temperature on growth, sclerotia, aflatoxins and aflD $(=$ nor-1) expression by Aspergillus flavus RCP08108. Microbiol. Res. 167, 470-477. doi: 10.1016/j.micres.2011. 11.004

Paul, S., Dubey, R. C., Maheswari, D. K., and Kang, S. C. (2011). Trachyspermum ammi (L.) fruit essential oil influencing on membrane permeability and surface characteristics in inhibiting food-borne pathogens. Food Control 22, 725-731. doi: 10.1016/j.foodcont.2010.11.003

Proctor, R. H., Brown, D. W., Plattner, R. D., and Desjardins, A. E. (2003). Co-expression of 15 contiguous genes delineates a fumonisin biosynthetic gene cluster in Gibberella moniliformis. Fungal Genet. Biol. 38, 237-249. doi: 10.1016/S1087-1845(02)00525-X

Samapundo, S., Devliehgere, F., De Meulenaer, B., and Debevere, J. (2005). Effect of water activity and temperature on growth and the relationship between fumonisin production and the radial growth of Fusarium verticillioides and Fusarium proliferatum on corn. J. Food Protect. 68, 1054-1059.

Seefelde, W. G. M., and Humpf, H. U. (2002). Analysis of fumonisin B1 in Fusarium proliferatum-infected asparagus spears and garlic bulbs from Germany by liquid chromatography-Electrospray ionization mass spectrometry. J. Agric. Food Chem. 50, 2778-2781. doi: 10.1021/jf011 5037

Senyuva, H. Z., and Gilbert, J. (2008). Identification of fumonisin B2, HT-2 toxin, patulin and zearalenone in dried figs by liquid chromatography-time-of-flight mass spectrometry and liquid chromatography-mass spectrometry. J. Food Protect. 71, 1500-1504.

Seo, J. A., Proctor, R. H., and Plattner, R. D. (2001). Characterization of four clustered and coregulated genes associated with fumonisin biosynthesis in Fusarium verticillioides. Fungal Genet. Biol. 34, 155-165. doi: 10.1006/fgbi.2001.1299

Susca, A., Proctor, R. H., Mule, G., Stea, G., Ritieni, A., Logrieco, A., et al. (2010). Correlation of mycotoxin fumonisin B-2 production and presence of the fumonisin biosynthetic gene fum8 in Aspergillus niger from grape. J. Agric. Food Chem. 58, 9266-9272. doi: 10.1021/jf101591x

Tao, N., OuYang, Q., and Jia, L. (2014). Citral inhibits mycelial growth of Penicillium italicum by a membrane damage mechanism. Food Control 41, 116-121. doi: 10.1016/j.foodcont.2014.01.010

Torres, A. M., Ramirez, M. L., Arroyo, M., Chulze, S. N., and Magan, N. (2003). Potential use of antioxidants for control of growth and fumonisin production by Fusarium verticillioides and Fusarium proliferatum on whole maize grain. Inter. J. Food Microbiol. 83, 319-324. doi: 10.1016/S0168-1605(02) 00380-X

Velluti, A., Sanchis, V., Ramos, A. J., Egido, J., and Marin, S. (2003). Inhibitory effect of cinnamon, clove, lemongrass, oregano and palmarose essential oils on growth and fumonisin B-1 production by Fusarium proliferatum in maize grain. Inter. J. Food Microbiol. 89, 145-154. doi: 10.1016/S0168-1605(03) 00116-8

Xing, F., Hua, H., Selvaraj, J. N., Yuan, Y., Zhao, Y., Zhou, L., et al. (2014a). Degradation of fumonisin B1 by cinnamon essential oil. Food Control 38, 37-40. doi: 10.1016/j.foodcont.2013.09.045 
Xing, F., Hua, H., Selvaraj, J. N., Zhao, Y., Zhou, L., Liu, X., et al. (2014b). Growth inhibition and morphological alterations of Fusarium verticillioides by cinnamon oil and cinnamaldehyde. Food Control 46, 343-350. doi: 10.1016/j.foodcont.2014.04.037

Yang, J., Li, J., Jiang, Y., Duan, X., Qu, H., Yang, B., et al. (2014). Natural occurrence, analysis, and prevention of mycotoxins in fruits and their processed products. Crit. Rev. Food Sci. Nutr. 54, 64-83. doi: 10.1080/10408398.2011. 569860

Zhan, R. L., Yang, S. J., Ho, H. H., Liu, F., Zhao, Y. L, Chang, J. M., et al. (2010). Mango malformation disease in south china caused by Fusarium proliferatum. J. Phytopathol. 158, 721-725. doi: 10.1111/j.1439-0434.2010.01688.x
Conflict of Interest Statement: The authors declare that the research was conducted in the absence of any commercial or financial relationships that could be construed as a potential conflict of interest.

Copyright (c) 2016 Li, Jian, Chen, Wang, Gong, Duan, Yang and Jiang. This is an open-access article distributed under the terms of the Creative Commons Attribution License (CC BY). The use, distribution or reproduction in other forums is permitted, provided the original author(s) or licensor are credited and that the original publication in this journal is cited, in accordance with accepted academic practice. No use, distribution or reproduction is permitted which does not comply with these terms. 